Cite this: Phys. Chem. Chem. Phys., 2012, 14, 8974-8980

\title{
Water-wire catalysis in photoinduced acid-base reactions
}

\author{
Oh-Hoon Kwon* and Omar F. Mohammed* \\ Received 29th November 2011, Accepted 1st February 2012 \\ DOI: $10.1039 / \mathrm{c} 2 \mathrm{cp} 23796 \mathrm{~b}$
}

The pronounced ability of water to form a hyperdense hydrogen $(\mathrm{H})$-bond network among itself is at the heart of its exceptional properties. Due to the unique H-bonding capability and amphoteric nature, water is not only a passive medium, but also behaves as an active participant in many chemical and biological reactions. Here, we reveal the catalytic role of a short water wire, composed of two (or three) water molecules, in model aqueous acid-base reactions synthesizing 7-hydroxyquinoline derivatives. Utilizing femtosecond-resolved fluorescence spectroscopy, we tracked the trajectories of excited-state proton transfer and discovered that proton hopping along the water wire accomplishes the reaction more efficiently compared to the transfer occurring with bulk water clusters. Our finding suggests that the directionality of the proton movements along the charge-gradient H-bond network may be a key element for long-distance proton translocation in biological systems, as the H-bond networks wiring acidic and basic sites distal to each other can provide a shortcut for a proton in searching a global minimum on a complex energy landscape to its destination.

\section{Introduction}

Water is a fundamental medium to many chemical and biological processes. ${ }^{1-3}$ The classical role of water has been regarded as a dielectric solvent, which affects energetics of molecules, accelerating or decelerating certain reactions involved. Recently, the importance of ubiquitous water not only as a nonspecific, passive dielectric medium, but also as an active participant in reactions has been pointed out. ${ }^{2}$ A single water molecule has been reported to function as a catalyst in chemical reactions. ${ }^{4-6}$ In more complicated biological reactions in, e.g., cytochrome c oxidase, purple membrane proteins, green fluorescent proteins, or some enzymes, hydrogen $(\mathrm{H})$-bond bridges involving water molecules (water wires) have been suggested to be essential for the proton conduction von Grotthuss mechanism. ${ }^{6-9}$ However, due to the complicated feature of proteinous systems, mechanistic understanding of the role of water H-bond networks in reaction dynamics still remains to be elucidated at a molecular level.

Proton-transfer reactions in aqueous solutions may involve different trajectories and mechanisms, depending on the reactivities of acids and bases, the configuration of $\mathrm{H}$-bond networks, the number of water molecules between acid and base, and solvent fluctuations. ${ }^{10-20}$ In this regard, the proton translocation in bifunctional heteroaromatic molecules, featuring both proton donor and acceptor groups distal to each other, can serve as a simplest model reaction to experimentally examine the

Physical Biology Center for Ultrafast Science and Technology, Arthur Amos Noyes Laboratory of Chemical Physics,

California Institute of Technology, Pasadena, CA 91125, USA.

E-mail: ohkwon@caltech.edu,omar3070@caltech.edu catalytic function of water wires. ${ }^{17-20}$ 7-Hydroxyquinoline (7HQ) and related probe molecules are excellent candidates among those because proton donating and accepting groups are at well-defined positions forming proton-transfer coordinates. ${ }^{21-26}$

Upon photoexcitation to the first excited singlet state $\left(\mathrm{S}_{1}\right)$, the enol and the imine groups of $7 \mathrm{HQ}$ become more acidic and basic, respectively, relative to those in the ground state, driving excited-state proton transfer to occur. ${ }^{21}$ Two different isomers of cis-7HQ and trans-7HQ, depending on the orientation of the enol group with respect to the imine group, can exist (Chart 1). ${ }^{25-27}$ The cis-7HQ in both polar aprotic and nonpolar solvents can form a cyclically H-bonded complex with protic guest molecules, with which proton relay from the acidic to the basic site of the probe molecule takes place, resulting in the production of a keto tautomer. ${ }^{23}$ In protic solvents, however, the presence of both rotamers and the possible interruption of $\mathrm{H}$-bond networks in the cyclically $\mathrm{H}$-bonded
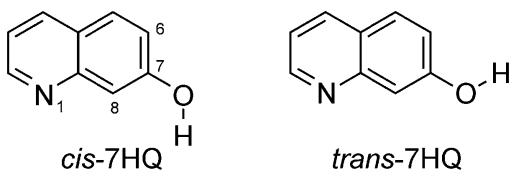<smiles>[1H]Cc1cc2cccnc2cc1O</smiles><smiles>[1H][CH]c1ccc2cccnc2c1O</smiles>

$6 \mathrm{Me}-7 \mathrm{HQ}$

\section{$8 \mathrm{Me}-7 \mathrm{HQ}$}

Chart 1 Structures of probe molecules. 
(a)<smiles></smiles>

$\left(\mathrm{H}_{2} \mathrm{O}\right)_{m}$

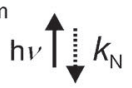

(b)

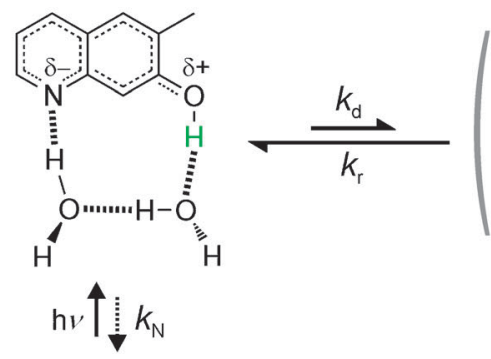

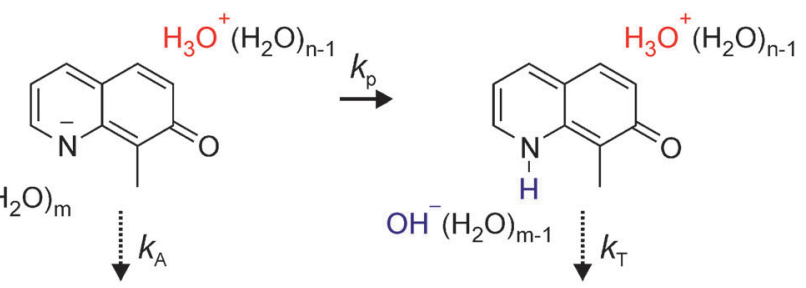
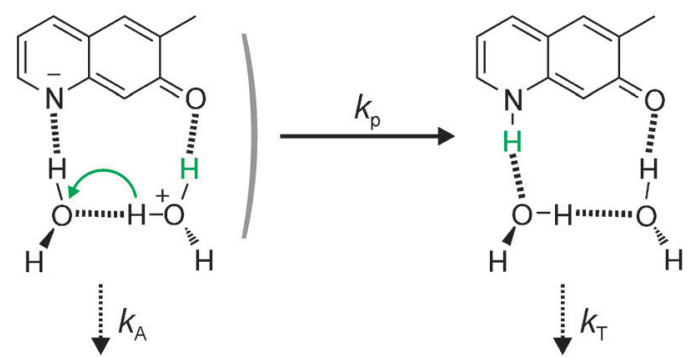

Scheme 1 Tautomerization pathways in water. (a) 8Me7HQ: because a methyl group at the 8 position hinders the formation of a water wire linking the acidic enol and the basic imine groups of the probe molecule, independent water clusters around each functional group must participate in the acid-base reaction. (b) 6Me7HQ: by introducing the steric imposition at the 6 position, a cyclically H-bonded complex can preferentially form with a water wire, which can catalyze the reaction. $k_{\mathrm{d}}, k_{\mathrm{r}}$, and $k_{\mathrm{p}}$ are rates for the deprotonation of the enol group, its reprotonation, and the protonation of the imine group, respectively. $k_{\mathrm{N}}, k_{\mathrm{A}}$, and $k_{\mathrm{T}}$ denote the relaxation rates of $\mathrm{N}^{*}, \mathrm{~A}^{*}$, and $\mathrm{T}^{*}$, respectively, at $\mathrm{S}_{1}$ in radiative and nonradiative ways.

complex can complicate observations and, thus, interpretation of the prototropic tautomerization dynamics. ${ }^{24-26}$

To decipher the catalytic roles of water wires in aqueous acid-base reactions, it is desired to reduce such complexities. Here, we have carefully designed two chemical probes, 8-methyl-7HQ (8Me7HQ) and 6-methyl-7HQ (6Me7HQ), as a model system to study proton conduction in water (Chart 1). The former has the enol group in the trans configuration and cannot undergo proton relay along a water wire because a methyl group at the 8 position hampers the formation of the cyclically H-bonded complex with water molecules. ${ }^{25}$ In this case, the two prototropic groups are hydrated independently and only intermolecular acid-base reactions are suggested to occur with independent water clusters around each functional group (see Scheme 1). In contrast, steric hindrance at the 6 position imposes preferential formation of the cis rotamer. As such, the molecular design of 6Me7HQ can favor the formation of a cyclically $\mathrm{H}$-bonded complex with a water wire, which catalyzes proton translocation from the acidic group to the basic group.

In this study, we dissect ultrafast aqueous acid-base reactions revealing the catalytic behavior of water wires, with the aid of femtosecond (fs)-resolved fluorescence spectroscopy. Mechanistic details on the proton conduction along the H-bonding water wires are presented.

\section{Experimental section}

6Me7HQ and 8Me7HQ were synthesized according to the conventional Skraup reaction following the procedure previously reported, ${ }^{25}$ and subsequently purified twice by column chromatography. 7HQ (99\%) was purchased from Across and used without further purification. The perdeuterated solvent $\mathrm{D}_{2} \mathrm{O}$ was obtained from Sigma-Aldrich and used as received. The $\mathrm{pH}$ and $\mathrm{pD}$ of aqueous solutions were adjusted by adding $\mathrm{HCl} / \mathrm{NaOH}$ and $\mathrm{DCl} / \mathrm{NaOD}$ solutions, respectively. All the
$\mathrm{pD}$ values were corrected from $\mathrm{pH}$-meter readings. The concentration of samples was $1 \times 10^{-4} \mathrm{M}$ for steady-state measurements and time-correlated single-photon counting (TCSPC) measurements, while it was $5 \times 10^{-4} \mathrm{M}$ for fs-resolved fluorescence up-conversion measurements.

Static absorption spectra were recorded by using a Cary 50 spectrometer (Varian) with $10 \mathrm{~mm}$ quartz cells. Steady-state fluorescence spectra were measured using a FluoroMax-2 fluorimeter (ISA-Spex) with $10 \mathrm{~mm}$ rectangular quartz cells. The fluorescence spectra were collected in a right-angle geometry and corrected for spectral sensitivity of the instrument.

fs-Resolved transients were obtained using the fluorescence up-conversion technique. The output of an amplified Ti-sapphire laser system (Spectra-Physics, Hurricane X) produced $100 \mathrm{fs}$ pulses centered at $800 \mathrm{~nm}$ (fundamental) at a repetition rate of $1 \mathrm{kHz}$ and energy of $0.8 \mathrm{~mJ}$. All fs-resolved transients were obtained by the excitation of samples at $330 \mathrm{~nm}$, which is achieved through an optical parametric amplifier (SpectraPhysics, OPA-800C) and a combination of nonlinear crystals. Excitation energy was attenuated to $200 \mathrm{~nJ}$. The up-converted fluorescence signal was taken at the magic angle $\left(54.7^{\circ}\right)$ of the pump polarization relative to the gate polarization, parallel to the acceptance axis of the up-conversion crystal, to eliminate the influence of induced sample anisotropy on the signal. The temporal response of the instrument was found to be $330 \mathrm{fs}$. The fluorescence transients were fit to theoretical functions, using the IGOR Pro program (WaveMetrics) for the convolution of the Gaussian instrument response function with a sum of exponential functions.

Fluorescence lifetimes longer than 500 ps were measured by utilizing a TCSPC spectrometer (FluoTime 200, PicoQuant) coupled to a broad-band tunable Ti-sapphire oscillator (MaiTai HP, Spectra-Physics) as an excitation source. The repetition rate of excitation was attenuated to $8 \mathrm{MHz}$ using a pulse picker (Model 3980-5, Spectra-Physics). Desired excitation wavelengths 
were achieved by the frequency-doubling or -tripling of tunable fundamental wavelength $(700-1020 \mathrm{~nm})$. No sample degradation was observed through the measurements. All the experiments were performed at room temperature $\left(24 \pm 1^{\circ} \mathrm{C}\right)$. Further details of the setup are described elsewhere. ${ }^{28}$

\section{Results and discussion}

\section{Steady-state spectroscopy}

7HQ in water can adopt four prototropic forms depending on $\mathrm{pH}$ : a normal form (N), an enol-deprotonated anion (A), an imine-protonated cation $(\mathrm{C})$, and a tautomer $(\mathrm{T}) .^{21,24}$ For the ground-state equilibria, the $\mathrm{p} K_{\mathrm{a}}$ values of $\mathrm{C}$ and $\mathrm{N}$ have been reported to be 5.4 and 9.0, respectively. ${ }^{21,25}$ At pH 7.2, 7HQ exists exclusively as N (67\%) and T (29\%) in water. In Fig. 1, the absorption spectra of 7HQ and its derivatives at neutral $\mathrm{pH}$ are displayed to show two bands for $\mathrm{N}$ and $\mathrm{T}$ at around 325 and $410 \mathrm{~nm}$, respectively. Note that the lowest absorption bands of $\mathrm{A}$ and $\mathrm{C}$ at $\mathrm{pH} 12$ and 3, respectively, are identified to reside at around 345 and $370 \mathrm{~nm}$ and their contributions at neutral $\mathrm{pH}$ are negligible. ${ }^{21}$ The $\mathrm{p} K_{\mathrm{a}}$ values of $\mathrm{C}$ and $\mathrm{N}$ for $8 \mathrm{Me} 7 \mathrm{HQ}$ have been reported to be 5.6 and 9.5 , respectively, while those for $6 \mathrm{Me} 7 \mathrm{HQ}$ to be 5.3 and 9.2, respectively. ${ }^{25}$

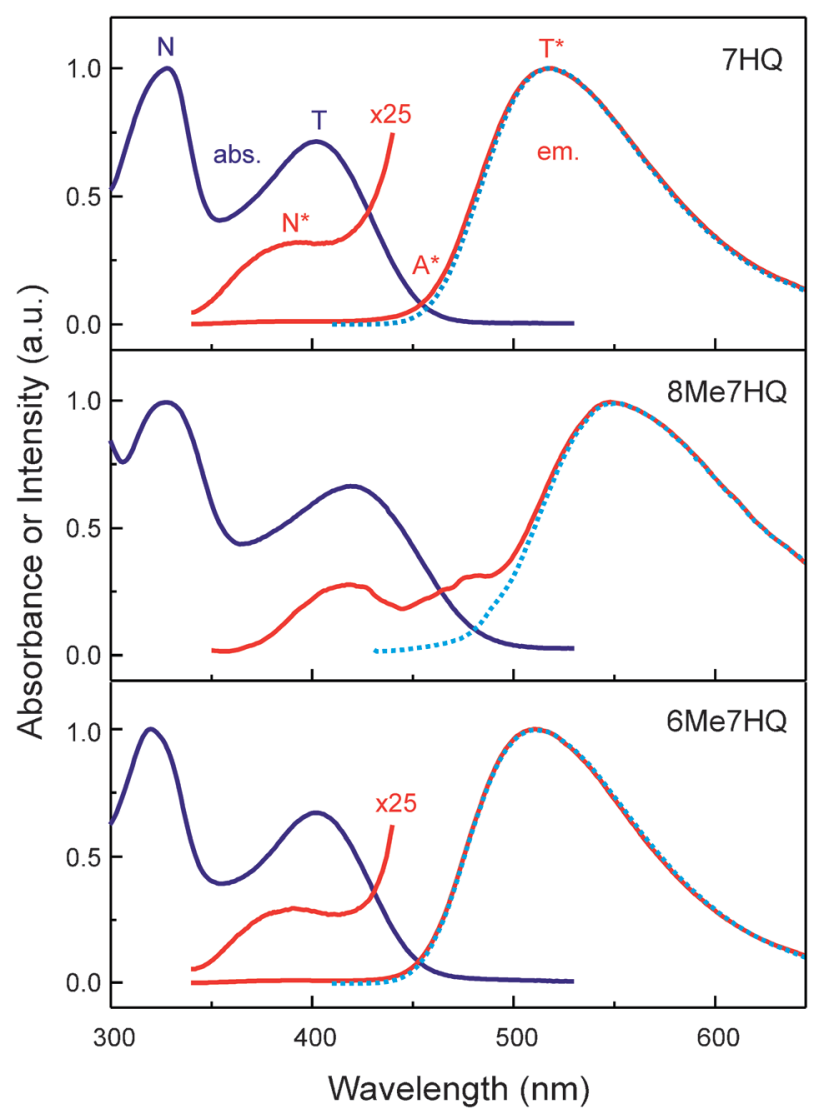

Fig. 1 Steady-state absorption and fluorescence spectra. pHs chosen for aqueous $7 \mathrm{HQ}, 8 \mathrm{Me} 7 \mathrm{HQ}$, and $6 \mathrm{Me} 7 \mathrm{HQ}$ are $7.1 \pm 0.1,7.4 \pm 0.1$, and $7.2 \pm 0.1$, respectively. Fluorescence spectra were recorded with excitation at 330 (solid) and $400 \mathrm{~nm}$ (dotted). At a steady state, tautomer emission $(\geq 500 \mathrm{~nm})$ prevails and the emission of parent molecules is evident at $\sim 400 \mathrm{~nm}$.
The excitation of 7HQ at $330 \mathrm{~nm}$ promotes $\mathrm{N}$ to its $\mathrm{S}_{1}$ state $\left(\mathrm{N}^{*}\right)$. This gives a fluorescence spectrum showing two main bands around 390 and $510 \mathrm{~nm}$, which are attributed to $\mathrm{N}^{*}$ and $\mathrm{T}^{*}$, of which the precursor is $\mathrm{N}^{*}$, respectively. ${ }^{21,23-26} \mathrm{~T}^{*}$ can also directly populate from the ground state upon excitation at $330 \mathrm{~nm}$, but its contribution to the steady-state spectrum is not significant and can be precisely determined from time-resolved measurements (see below). The difference at around $470 \mathrm{~nm}$ between the fluorescence spectrum with the excitation of $\mathrm{N}$ at $330 \mathrm{~nm}$ and that with the Franck-Condon excitation of $\mathrm{T}$ at $400 \mathrm{~nm}$ hints the existence of short-lived intermediate fluorescence. $^{24}$

The fluorescence spectrum of $8 \mathrm{Me} 7 \mathrm{HQ}$ also shows the two distinct bands of $\mathrm{N}^{*}$ and $\mathrm{T}^{*}$ at 415 and $540 \mathrm{~nm}$, respectively, as well as an evident intermediate band at around $490 \mathrm{~nm}$. On the other hand, the fluorescence spectrum of $6 \mathrm{Me} 7 \mathrm{HQ}$ shows that the intermediate band is greatly suppressed. The lack of intermediate fluorescence, as opposed to $8 \mathrm{Me} 7 \mathrm{HQ}$ and $7 \mathrm{HQ}$, is attributed to an altered tautomerization mechanism depending on the H-bonding environment around as revealed by ultrafast dynamics measurements (see below).

\section{Dynamics of 8Me7HQ}

Fig. 2 shows three representative fluorescence kinetic profiles of $8 \mathrm{Me} 7 \mathrm{HQ}$ with the excitation at $330 \mathrm{~nm}$ in neutral water: $\mathrm{N}^{*}$ at $415 \mathrm{~nm}$; an intermediate species at $490 \mathrm{~nm}$; and $\mathrm{T}^{*}$ at $580 \mathrm{~nm}$. $\mathrm{N}^{*}$ decays biexponentially in $3.2 \pm 0.3 \mathrm{ps}(32 \%)$ and $91 \pm 3 \mathrm{ps}$ $(68 \%)$. At $385 \mathrm{~nm}$, a blue wavelength with respect to the peak of $\mathrm{N}^{*}$ fluorescence at $415 \mathrm{~nm}$, an extra $820 \mathrm{fs}$ component $(64 \%)$

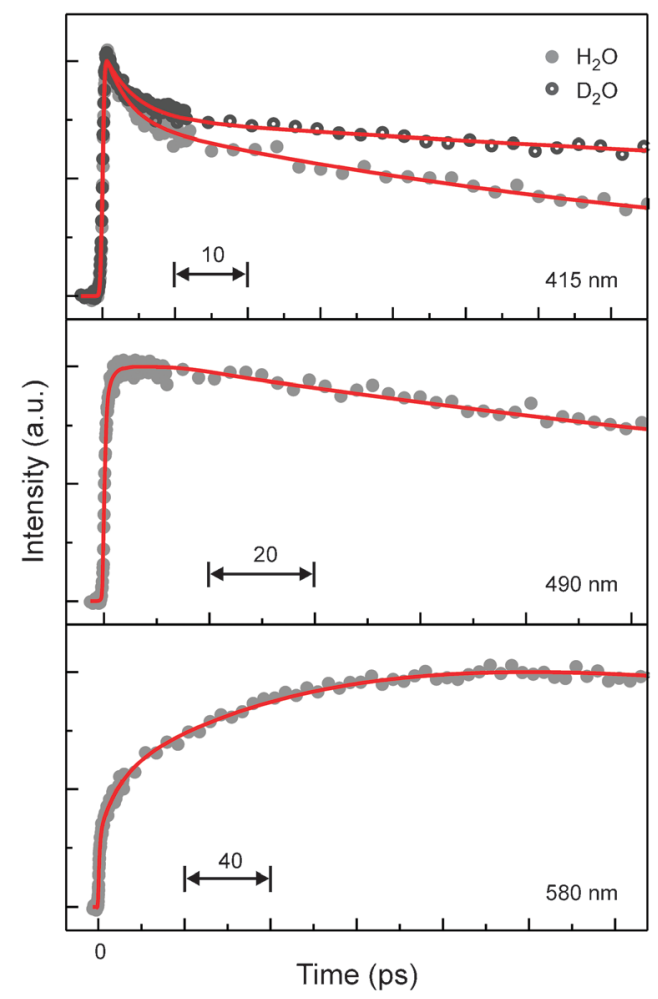

Fig. 2 Dynamics of 8Me7HQ: fluorescence transients of $8 \mathrm{Me} 7 \mathrm{HQ}$ in neutral water ( $\mathrm{pH} 7.4)$ were obtained following excitation at $330 \mathrm{~nm}$. The monitored wavelengths and multiexponential fits are given in each panel. 
was also detected. This ultrafast component is absent at $415 \mathrm{~nm}$ and is ascribed to solvation. The ratio of the $3.2 \mathrm{ps}$ component to the $\sim 100 \mathrm{ps}$ one is robust at both the wavelengths, indicating that $3.2 \mathrm{ps}$ is indeed the timescale of proton transfer. The majority of $\mathrm{T}^{*}$ rises in $109 \pm 5 \mathrm{ps}$ and the minor fraction $(9 \%)$, which forms within our instrumental temporal response, is ascribed to the Franck-Condon excitation of T (see above). The decay time of $\mathrm{T}^{*}$ is obtained to be $630 \mathrm{ps,}$ which is attributed to the lifetime of $\mathrm{T}^{*}$. Although the $\mathrm{N}^{*}$ and $\mathrm{T}^{*}$ fluorescence contribute to the fluorescence transient at $490 \mathrm{~nm}$ due to spectral congestion, it is found that the intermediate species rises in $3.4 \pm 0.3 \mathrm{ps}$ and decays in $130 \pm 20 \mathrm{ps}(40 \%)$, indicating that the intermediate is kinetically coupled to $\mathrm{N}^{*}$ and $\mathrm{T}^{*}$. Note that the significant fraction $(68 \%)$ of the $\sim 100$ ps component in the $\mathrm{N}^{*}$ fluorescence is a signature of the establishment of an equilibrium between $\mathrm{N}^{*}$ and the intermediate.

$8 \mathrm{Me} 7 \mathrm{HQ}$ has the enol group in a trans form and is unlikely to undergo proton hopping along a water wire. Because of the nature of $\mathrm{H}$-bonds with directionality and well-defined length, the methyl group at the 8 position hinders the formation of a cyclically H-bonded complex with a bridging water wire. From the finding that there are three global lifetimes, i.e., $3.2 \mathrm{ps}, 100 \mathrm{ps}$, and $630 \mathrm{ps}$, spanning the whole fluorescence spectrum, in which the lifetime of $\mathrm{T}^{*}(630 \mathrm{ps})$ is not seen at the $\mathrm{N}^{*}$ band and the blue side of the intermediate band, the simplest mechanism is proposed to be a two-step intermolecular acid-base reaction as depicted in Scheme 1a. In this scheme, the first step is reversible whereas the second step is irreversible. This can be translated into the following differential equation:

$$
\frac{\mathrm{d}}{\mathrm{d} t}\left[\begin{array}{l}
\mathrm{N}^{*} \\
\mathrm{~A}^{*} \\
\mathrm{~T}^{*}
\end{array}\right]=\left[\begin{array}{ccc}
-k_{\mathrm{d}}-k_{\mathrm{N}} & k_{\mathrm{r}} & 0 \\
k_{\mathrm{d}} & -k_{\mathrm{r}}-k_{\mathrm{p}}-k_{\mathrm{A}} & 0 \\
0 & k_{\mathrm{p}} & -k_{\mathrm{T}}
\end{array}\right] \times\left[\begin{array}{l}
\mathrm{N}^{*} \\
\mathrm{~A}^{*} \\
\mathrm{~T}^{*}
\end{array}\right]
$$

where $k_{\mathrm{d}}, k_{\mathrm{r}}$, and $k_{\mathrm{p}}$ refer to rates for the deprotonation of the enol group, its reprotonation, and the protonation of the imine group, respectively. $k_{\mathrm{N}}, k_{\mathrm{A}}$, and $k_{\mathrm{T}}$ are the relaxation rates of $\mathrm{N}^{*}$, $\mathrm{A}^{*}$, and $\mathrm{T}^{*}$, respectively, to their ground states, and $k_{\mathrm{N}}$ and $k_{\mathrm{A}}$ are taken to be $3 \times 10^{8} \mathrm{~s}^{-1}$ and $1 \times 10^{8} \mathrm{~s}^{-1}$, respectively. ${ }^{24} k_{\mathrm{T}}$ is the reciprocal of the $\mathrm{T}^{*}$ lifetime. From the rate equation, $k_{\mathrm{d}}, k_{\mathrm{r}}$, and $k_{\mathrm{p}}$ are deduced to be $(9.4 \pm 1.0 \mathrm{ps})^{-1},(5.3 \pm 0.5 \mathrm{ps})^{-1}$, and $(35 \pm 4 \mathrm{ps})^{-1}$, respectively. Likewise, in $\mathrm{D}_{2} \mathrm{O}, k_{\mathrm{d}}, k_{\mathrm{r}}$, and $k_{\mathrm{p}}$ are obtained to be $(13.4 \pm 1.4 \mathrm{ps})^{-1},(6.6 \pm 0.7 \mathrm{ps})^{-1}$, and $(128 \pm 13 \mathrm{ps})^{-1}$, respectively.

At neutral $\mathrm{pH}$, the concentrations of hydronium and hydroxide ions are too low to participate in acid-base reactions at $\mathrm{S}_{1}$. Because typical diffusion-limited bimolecular rate constants for hydroxide and hydronium ions are $1 \times 10^{10}-1 \times 10^{11} \mathrm{M}^{-1} \mathrm{~s}^{-1}$, the timescales of the reactions must be on hundreds of $\mu$ s to ms. Therefore, on the timescale spanning ps and ns, the acid-base neutralization should occur to/from neutral water clusters. At $\mathrm{pH}$ 12 , the lifetime of $\mathrm{A}^{*}$, which captures a proton from a water cluster to generate $\mathrm{T}^{*}$, is obtained to be $160 \mathrm{ps}$, similar to that of the intermediate species ( $\sim 100 \mathrm{ps})$. At $\mathrm{pH} 2$, the lifetime of $\mathrm{C}^{*}$, which releases a proton to water clusters, is measured to be $\leq 10$ ps (data not shown). Accordingly, the identity of the intermediate is confirmed to be $\mathrm{A}^{*}$.

The formation of $\mathrm{A}^{*}$ as an exclusive reaction intermediate can be rationalized in the frame of intrinsic electronic energetics of each prototropic species involved. The intermolecular proton-transfer rates are closely related to the excess stabilization energies of conjugate acid and base that render proton transfer to be highly exothermic and facile. ${ }^{12,13}$ The lowest electronic-transition energy of $\mathrm{A}^{*}$ is deduced to be smaller than that of $\mathrm{C}^{*}$ from the lowest-absorption spectral position of A $\left(27027 \mathrm{~cm}^{-1}\right)$ and $\mathrm{C}\left(28571 \mathrm{~cm}^{-1}\right)$. This indicates that the deprotonation of $\mathrm{N}^{*}$ is more exoergic than the protonation, forming $\mathrm{A}^{*}$ as an exclusive reaction intermediate.

The kinetic isotope effect (KIE) of $1.4 \pm 0.2$ for the ultrafast enol deprotonation of $\mathrm{N}^{*}\left(k_{\mathrm{d}}\right)$ to a water cluster nearby and a relatively large KIE $(3.4 \pm 0.5)$ for the slower imine protonation of $\mathrm{A}^{*}\left(k_{\mathrm{p}}\right)$ are in line with the free-energy relationship; the more the reaction is exothermic, the smaller the KIE because the reaction rate approaches the asymptotic value for isotope-insensitive solvent motions on the timescale of a few ps. ${ }^{30}$ The large KIE of imine protonation supports the idea that the main origin of the proton is not from diffusion but from a contact neutral water cluster which has weaker acidity. It follows that the ejected proton from the enol group of 8Me7HQ diffuses into bulk water, resulting in stepwise tautomerization via the formation of transient intermediate $\mathrm{A}^{*}$.

\section{Dynamics of 6Me7HQ}

6Me7HQ exists mainly in the cis form due to the steric enforcement by a methyl group at the 6 position (see Scheme 1b). Consequently, it is facilitated to form a cyclically H-bonded complex with two (or three) water molecules, relaying a proton from the enol to the imine group. ${ }^{31}$ For the tautomerization of cis-7HQ in polar aprotic solvents, two to three water molecules have been reported to form a bridging H-bond wire. ${ }^{23 b, 31}$ In the gas phase two to three water molecules are experimentally and theoretically found to form a cyclically H-bonded complex with $7 \mathrm{HQ}^{32,33}$

$\mathrm{N}^{*}$ fluorescence at $385 \mathrm{~nm}$ shows a biphasic decay composed of $21 \pm 1(71 \%)$ and $2.3 \pm 0.2$ ps (Fig. 3). The 2.3 ps decay (29\%) still exists at $415 \mathrm{~nm}$, a red wavelength with respect to the peak of $\mathrm{N}^{*}$ fluorescence, and this component cannot be that of solvation. $\mathrm{T}^{*}$ fluorescence at $580 \mathrm{~nm}$ rises in $20 \pm 1$ ps $(52 \%)$ and decays in $3.2 \mathrm{~ns} ; 12 \%$ of $\mathrm{T}^{*}$ forms in $84 \pm 10$ ps. At $470 \mathrm{~nm}$, presumably a representative wavelength of intermediate $\mathrm{A}^{*}$, the fluorescence transient is almost flat over the early time window. The dynamics is dominated by the long lifetime of $\mathrm{T}^{*}(66 \%)$ with its $20 \mathrm{ps}$ rise being almost cancelled by the $\mathrm{N}^{*}$ decay. The transient also bears minor decay components, $102 \pm 26$ ps $(28 \%)$ and $\sim 2$ ps $(6 \%)$.

As dictated by the molecular design, there clearly emerges a new prevalent time constant of $20 \mathrm{ps}$ for the concomitant population change of $\mathrm{N}^{*}$ and $\mathrm{T}^{*}(\mathrm{KIE}=3.8 \pm 0.2) .2$ and $\sim 90$ ps components (see Table 1), which are similar to those for the case of $8 \mathrm{Me} 7 \mathrm{HQ}$, are found to exist as minor ones. This complex dynamic behavior of $6 \mathrm{Me} 7 \mathrm{HQ}$ is attributed to the bifurcation of the reaction pathway possibly depending on two different hydration environments around $6 \mathrm{Me} 7 \mathrm{HQ}$ in the ground state at the moment of excitation. Because the population changes of $\mathrm{N}^{*}$ and $\mathrm{T}^{*}$ are kinetically coupled on the time scale of $20 \mathrm{ps}$, we ascribe the $71 \%$ of $6 \mathrm{Me} 7 \mathrm{HQ}$ to cyclically H-bonded complexes with water molecules in the form of wires. These complexes undergo tautomerization via a different reaction pathway from that of $8 \mathrm{Me} 7 \mathrm{HQ}$ without building up the intermediate 


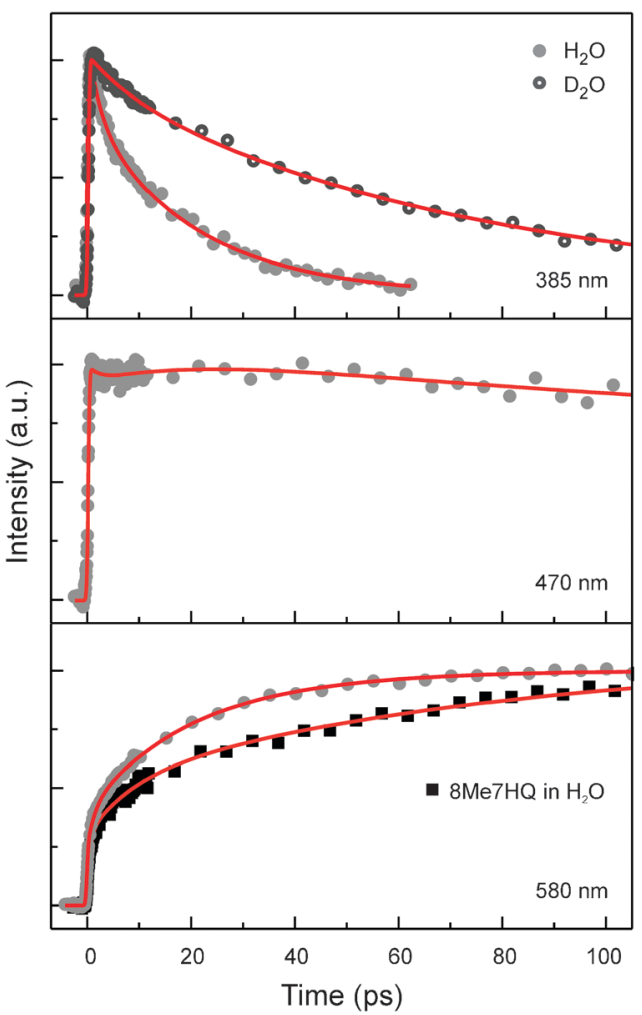

Fig. 3 Dynamics of 6Me7HQ: fluorescence transients of 6Me7HQ in neutral water $(\mathrm{pH} 7.2)$ were obtained following excitation at $330 \mathrm{~nm}$. The monitored wavelengths and multiexponential fits are given in each panel. For comparison, the transient of $8 \mathrm{Me} 7 \mathrm{HQ}$ at $580 \mathrm{~nm}$ is also depicted.

Table 1 Time constants to fit fluorescence kinetic profiles in Fig. 2 and 3

\begin{tabular}{|c|c|c|c|c|c|c|c|c|}
\hline \multirow[b]{3}{*}{ Rotamer } & \multirow[b]{3}{*}{ Solvent } & \multirow{3}{*}{$\begin{array}{l}\text { Wavelength }{ }^{a} / \\
\mathrm{nm}\end{array}$} & \multicolumn{6}{|c|}{ Time constant ${ }^{b} / \mathrm{ps}$} \\
\hline & & & \multicolumn{3}{|l|}{ Rise } & \multicolumn{3}{|c|}{ Decay } \\
\hline & & & $\tau_{1}$ & $\tau_{2}$ & $\tau_{3}$ & $\tau_{4}$ & $\tau_{5}$ & $\tau_{6}$ \\
\hline \multirow[t]{4}{*}{ 8Me7HQ (trans) } & $\mathrm{H}_{2} \mathrm{O}$ & 415 & & & & 3.2 & 91 & \\
\hline & & 490 & 0.49 & 3.4 & & 130 & 630 & \\
\hline & & 580 & 4.2 & 109 & & 630 & & \\
\hline & $\mathrm{D}_{2} \mathrm{O}$ & 415 & & & & 4.3 & 308 & \\
\hline \multirow[t]{4}{*}{ 6Me7HQ (cis) } & $\mathrm{H}_{2}^{2} \mathrm{O}$ & 385 & & & & 2.3 & 21 & \\
\hline & & 470 & 20 & & & 2.3 & 102 & 3200 \\
\hline & & 580 & 1.56 & 20 & 84 & 3200 & & \\
\hline & $\mathrm{D}_{2} \mathrm{O}$ & 385 & & & & 9.0 & 73 & \\
\hline
\end{tabular}

${ }^{a}$ Monitored fluorescence wavelength. ${ }^{b}$ Time constants greater than 500 ps are adopted from TCSPC measurements in this work.

species (Scheme 1b). This dynamic behavior explains the great suppression of the intermediate fluorescence of $6 \mathrm{Me} 7 \mathrm{HQ}$ at steady state compared to that of $8 \mathrm{Me} 7 \mathrm{HQ}$ (see Fig. 1).

The other $29 \%$ of $6 \mathrm{Me} 7 \mathrm{HQ}$, on the other hand, decays in $2.3 \pm 0.2 \mathrm{ps}$, which is similar to the fast decay time of $8 \mathrm{Me} 7 \mathrm{HQ}$ $(3.2 \pm 0.3 \mathrm{ps})$. The $\sim 100$ ps component of small population is also observed as a decay at $470 \mathrm{~nm}$ and a rise at $580 \mathrm{~nm}$. At $\mathrm{pH}=13$, the lifetime of the $\mathrm{A}^{*}$ form of $6 \mathrm{Me} 7 \mathrm{HQ}$ is obtained to be $150 \mathrm{ps}$. These kinetic similarities to $8 \mathrm{Me} 7 \mathrm{HQ}$ point out that $6 \mathrm{Me} 7 \mathrm{HQ}$ with two functional groups independently hydrated exists as a relatively small population and undergoes acid-base neutralization with water clusters under a bulk hydration environment. From the fraction of the cyclic complex, we deduce $\Delta G$ for the formation of the water-wired complex at the ground-state equilibrium in water to be $-0.5 \mathrm{kcal} \mathrm{mol}^{-1}$.

The initial enol deprotonation of $6 \mathrm{Me} 7 \mathrm{HQ}$ to a water molecule in the wire is found to be significantly slower than the deprotonation to a water cluster in bulk (20 ps vs. 2.3 ps). If there were a fast interconversion between the wired form and the cluster form of water, $\mathrm{N}^{*}$ would quickly transfer a proton to the adjacent water cluster to form the $\mathrm{A}^{*}$ intermediate in $2.3 \mathrm{ps}$. However, we find that the slower pathway is a dominant channel over the more facile pathway. This suggests that the water wire linking the acidic and basic groups is structurally stable enough to survive at least during the proton-transfer process. This can be rationalized in a way that the photoinduced delocalization of charge densities on the acidic and basic groups of a parent molecule involving $\mathrm{H}$-bond networks ${ }^{34}$ can induce tight $\mathrm{H}$-bonds along the wire, partially in place of $\mathrm{H}$-bonding interaction to surrounding bulk water. Accordingly, the water wire can be regarded as an integrated chemical part of the H-bonded molecular complex. This extended resonance character has been witnessed in the enhancement of oscillator strength in the electronic absorption spectra of $7 \mathrm{HQ}$ when it forms a cyclically H-bonded complex with protic guest molecules. $^{23}$

A key question about the tautomerization mechanism along the wire is whether it occurs in a stepwise or a concerted manner. In the case of $6 \mathrm{Me} 7 \mathrm{HQ}$ there are only $\mathrm{N}^{*}$ and $\mathrm{T}^{*}$ bands in the steady-state fluorescence spectrum (Fig. 1, bottom). Moreover, from the correlation between the $\mathrm{N}^{*}$ decay and the $\mathrm{T}^{*}$ rise, the reaction pathway should be either concerted from $\mathrm{N}^{*}$ directly to $\mathrm{T}^{*}$ or stepwise with the first step to form a transient intermediate being rate-limiting. For the latter, the subsequent second step is subjected to be unresolvable. In the concerted pathway, all the protons are synchronous and in flight at the same rate according to its strict definition. This seems unlikely the case for the proton translocation in 6Me7HQ because of the asymmetric nature of functional groups in acidity as is revealed with $8 \mathrm{Me} 7 \mathrm{HQ}$ and the many-body involvement. We note that a previous proton inventory study on 7HQ complexed with a water wire in polar aprotic environments has shown that the proton moves in an asynchronous manner. ${ }^{23 b, c}$

The short H-bonding water wire delocalizes high charge density on the enol and imine groups attenuating the acidity and the basicity of each functional group. As such, enol deprotonation and imine protonation can be retarded; a water molecule, in the wire and adjacent to the acidic enol group, possesses less activity than the cluster-form in acid-base reactions as well. On the other hand, the protonation of the imine group by a hydronium cation during the proton relay is expected to be faster than that by a neutral water cluster because of stronger acidity. The timescale of $20 \mathrm{ps}$ is observed to be slower than the deprotonation to a water cluster $\left[(9 \mathrm{ps})^{-1}\right]$ but faster than the protonation from another cluster $\left[(35 \mathrm{ps})^{-1}\right]$. Therefore, it is plausible to attribute the overall rate $\left[(20 \mathrm{ps})^{-1}\right]$ to that of deprotonation of the enol group $\left(k_{\mathrm{d}}\right)$ having weaker photoacidity.

The proton hopping along a water wire for 6Me7HQ occurs via the rate-determining deprotonation of the acidic enol group 
to produce a hydronium cation experiencing an activation barrier and KIE of 3.8. The hydrated proton is then transferred, in a von Grotthuss fashion through the H-bond networks of the water wire, to protonate the proximate imine group $\left(\mathrm{p} K_{\mathrm{b}} *=15.3\right){ }^{24 c}$ barrierless and in a rapid (presumably within several ps) step. For example, with a typical proton diffusion coefficient of $\sim 1.0 \times$ $10^{-4} \mathrm{~cm}^{2} \mathrm{~s}^{-1}$ it takes about 4 ps to travel $5 \AA$. The lifetime of a transient hydronium ion has been reported to be several ps in a proton-relay process involving a weaker acetate base of $\mathrm{p} K_{\mathrm{b}}=$ 9.25. ${ }^{14,15}$ The transient hydronium cation complexed with intermediate $\mathrm{A}^{*}$ may be effectively in the Zundel-like configuration, in which the proton is symmetrically shared by two water molecules, due to strong $\mathrm{H}$-bonds along the wire at the expense of the interaction with surrounding bulk water (Scheme 1). ${ }^{11,12}$ Essentially the above picture is in line with ultrafast infrared (IR) spectroscopic studies, in which sequential proton hopping is reported to take place for photoacid- $\left(\mathrm{H}_{2} \mathrm{O}\right)_{n}$-base systems. $^{14,15,35}$

\section{Bifurcation of proton-transfer pathway}

The trajectories of proton transfer depending on $\mathrm{H}$-bond networks in aqueous environments are given in Fig. 4. Upon electronic excitation, the extended charge delocalization involving the water wire stabilizes the cyclically H-bonded complex from the surrounding aqueous bath (Fig. 4b); in this sense, the wire is an integrated part of the chemical system. Although the initial enol deprotonation to the water wire is slower than that to a water cluster, the directionality of proton conduction along the charge-gradient wire, i.e., a "push-pull" effect, ${ }^{36}$ renders successive imine protonation to occur rapidly. This is in contrast to the accumulation of metastable A* as an intermediate in the stepwise pathway involving water clusters with imine protonation being rate limiting. The tautomerization catalyzed by the wire takes $20 \mathrm{ps}$, four times shorter than the lifetime of the intermediate in the reaction with water clusters. This signifies the catalytic role of a water wire in the prototropic reactions modulating free energies and barriers (see Fig. 4c).

It seems that the key factor to differentiate the proton transfer pathway between $6 \mathrm{Me} 7 \mathrm{HQ}$ and $8 \mathrm{Me} 7 \mathrm{HQ}$ lies in the number of water molecules connecting the acidic and the basic groups. In an aqueous photoacid-base system, it has been reported that the number of water molecules separating an acid and a base determines the acid-base neutralization mechanism. ${ }^{37}$ For configurations with acid-base separated by less than four water molecules, diffusion has been reported to play a negligible role in the reaction because these configurations are already "reactive complexes." Diffusion dominates the reaction dynamics when four or more water molecules separate the acid and the base. The present results are in agreement with the previous study in that the molecular design of $8 \mathrm{Me} 7 \mathrm{HQ}$ with a bulky methyl group at the 8 position exerts a steric hindrance to orient the acidic enol group farther away from the basic imine group. Furthermore, the position of the bulky group is in the middle of the proton-transfer coordinate and this ought to significantly increase the number of water molecules separating the acidic and basic groups to interrupt the formation of the reactive wire configuration.

Before concluding, we note that the bifurcation of tautomerization trajectory depending on the hydration environment
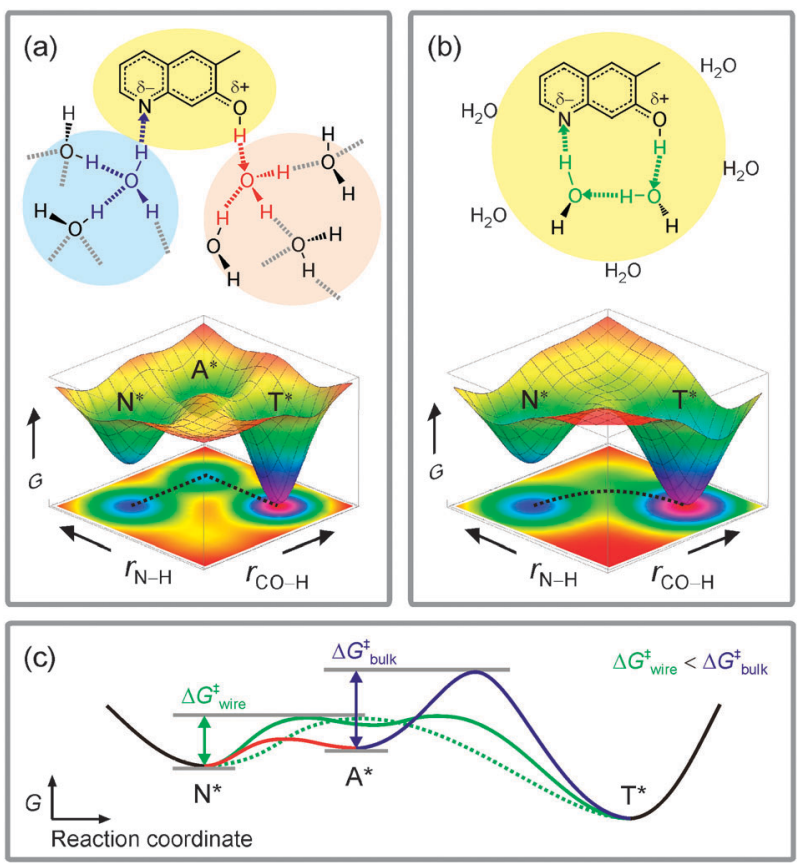

Fig. 4 Catalytic role of a water wire in prototropic tautomerization. (a) Schematic representation of bulk hydration for the acid-base reaction in water (top) and a pictorial free energy surface (bottom). Upon photoexcitation, electronic charge redistribution induces strong photoacidity and photobasicity on the enol and the imine group of the probe molecule, respectively, driving the reaction to occur. Independent water clusters hydrating the two functional groups are involved in the course of the reaction. The pictorial energy surface is constructed on the basis of understanding acquired in this study. Multiple minima depict $\mathrm{N}^{*}, \mathrm{~A}^{*}$, and $\mathrm{T}^{*}$ structures participating in the reaction. The intermediate $\mathrm{A}^{*}$ species can be mapped due to its metastable nature with the lifetime of $\sim 100$ ps. (b) Schematic representation of a cyclic $\mathrm{H}$-bond formation with a water wire, catalyzing the aqueous acid-base reaction (top), and the corresponding illustration of a free energy surface (bottom). When the proton-hopping mechanism via water-wire catalysis is operative, the tautomerization can be accelerated experiencing a lower rate-limiting activation barrier without building up intermediate species. (c) Comparison of energetics with and without the water-wire catalyst. $\Delta G^{\ddagger}$ is an activation barrier for the ratedetermining step in each reaction pathway (solid). Because of the unstable (short-lived) nature of $\mathrm{A}^{*}$ when complexed with a hydronium ion, the $A^{*}$ structure in the proton relay may be described as a transition state (dotted).

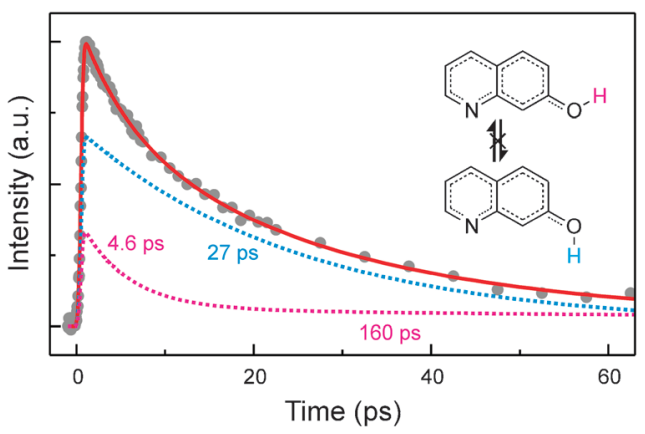

Fig. 5 Bifurcation of the reaction trajectory for 7HQ. Two prominent timescales (4.6 and $27 \mathrm{ps)}$ ) are evident in the fluorescence transient of $\mathrm{N}^{*}$ (probed at $395 \mathrm{~nm}$ ) in neutral water. Fits are deconvoluted according to two different tautomerization pathways (dotted). 
of precursors accommodates controversial observations made in the previous studies of aqueous acid-base neutralization of $7 \mathrm{HQ} .{ }^{24}$ In Fig. 5, the two prominent timescales are evident in the lifetime of $\mathrm{N}^{*}$. The bimodality can now be attributed to the coexistence of ground-state cis- and trans-rotamers, of which fractions are effectively frozen in $\mathrm{S}_{1}$ due to the increased double bond character of a $\mathrm{C}-\mathrm{O}$ bond, ${ }^{26,27}$ experiencing different hydration environments. This leads to branching off tautomerization into the proton relay along the water wire in $27 \pm 2 \mathrm{ps}^{38}$ and the acid-base reaction with independent water clusters forming the $\mathrm{A}^{*}$ intermediate, whose lifetime is $160 \mathrm{ps}$, in $4.6 \pm 0.5$ ps.

\section{Concluding remarks}

The findings made in this study indicate that the quasi 1-dimensional water wire is a more effective catalyst in the prototropic tautomerization reaction than bulk water. Although water can simultaneously solvate both cations and anions, that is vital for acid-base neutralization to occur, this unique amphoteric nature of water imposes the retardation of the reaction by stabilizing an ionic reaction intermediate. On the other hand, the strong $\mathrm{H}$-bond loop involving a complexed short water wire can confine a proton, dissociated from a photoacid and leads the proton to the eventual basic site. In this way, the reaction is efficiently accomplished without missing the ejected proton into the 3-dimensional H-bond network of bulk water. The perception discussed here of directional proton movements may provide fundamental knowledge for the understanding of a protonhopping mechanism in biological systems.

\section{Acknowledgements}

We are grateful to Prof. Ahmed H. Zewail for his persistent encouragement for the work and careful reading of the manuscript. We thank Prof. B. Stoltz for allowing access to his facilities for synthesis of chemicals and Dr. Sang Tae Park for reading of the manuscript. This work was supported by the National Science Foundation.

\section{References}

1 Y. Maréchal, The Hydrogen Bond and the Water Molecule: The Physics and Chemistry of Water, Aqueous and Biomedia, Elsevier, Amsterdam, 2007.

2 P. Ball, Chem. Rev., 2008, 108, 74.

3 P. Agre, Angew. Chem., Int. Ed., 2004, 43, 4278.

4 E. Vöhringer-Martinez, B. Hansmann, H. Hernandez-Soto, J. S. Francisco, J. Troe and B. Abel, Science, 2007, 315, 497.

5 Y. Matsuda, A. Yamada, K.-i. Hanaue, N. Mikami and A. Fujii, Angew. Chem., Int. Ed., 2010, 49, 4898.

6 Hydrogen-Transfer Reaction, ed. J. T. Hynes, J. P. Klinman, H.-H. Limbach and R. L. Schowen, Wiley-VCH, Weinheim, 2007, and references therein.

7 F. Garczarek and K. Gerwert, Nature, 2006, 439, 109.
8 C. Fang, R. R. Frontiera, R. Tran and R. A. Mathies, Nature, 2009, 462, 200.

9 J. Li, Z. Liu, C. Tan, X. Guo, L. Wang, A. Sancar and D. Zhong, Nature, 2010, 466, 887.

10 A. Douhal, F. Lahmani and A. H. Zewail, Chem. Phys., 1996, 207, 477.

11 G. A. Voth, Acc. Chem. Res., 2006, 39, 143.

12 N. Agmon, J. Phys. Chem. A, 2005, 109, 13.

13 L. M. Tolbert and K. M. Solntsev, Acc. Chem. Res., 2002, 35, 19.

14 (a) O. F. Mohammed, D. Pines, J. Dreyer, E. Pines and E. T. J. Nibbering, Science, 2005, 310, 83; (b) O. F. Mohammed, D. Pines, E. T. J. Nibbering and E. Pines, Angew. Chem., Int. Ed., 2007, 46, 1458.

15 M. J. Cox and H. J. Bakker, J. Chem. Phys., 2008, 128, 174501.

16 L. G. Arnaut and S. J. Formosinho, J. Photochem. Photobiol., A, 1993, 75, 1.

17 M. Kasha, J. Chem. Soc., Faraday Trans. 2, 1986, 82, 2379.

18 C.-C. Hsieh, C.-M. Jiang and P. T. Chou, Acc. Chem. Res., 2010, 43, 1364.

19 J. Waluk, Acc. Chem. Res., 2003, 36, 832.

20 M. Gutman and E. Nachliel, Annu. Rev. Phys. Chem., 1997, 48, 329.

21 S. F. Mason, J. Philp and B. E. Smith, J. Chem. Soc. A, 1968, 3051.

22 C. Tanner, C. Manca and S. Leutwyler, Science, 2003, 302, 1736.

23 (a) O.-H. Kwon, Y. S. Lee, B. K. Yoo and D.-J. Jang, Angew. Chem., Int. Ed., 2006, 45, 415; (b) S. Y. Park, Y. S. Lee, O.-H. Kwon and D.-J. Jang, Chem. Commun., 2009, 926; (c) S.-Y. Park, B. Kim, Y.-S. Lee, O.-H. Kwon and D.-J. Jang, Photochem. Photobiol. Sci., 2009, 8, 1611.

24 (a) E. Bardez, Isr. J. Chem., 1999, 39, 319; (b) S. I. Lee and D.-J. Jang, J. Phys. Chem., 1995, 99, 7537; (c) H. J. Park, O.-H. Kwon, C. S. Ah and D.-J. Jang, J. Phys. Chem. B, 2005, 109, 3938.

25 T. Nakagawa, S. Kohtani and M. Itoh, J. Am. Chem. Soc., 1995, 117, 7952 .

26 O.-H. Kwon, T. G. Kim, Y. S. Lee and D.-J. Jang, J. Phys. Chem. $B, 2006,110,11997$, and references therein.

27 F. Lahmani, A. Douhal, E. Breheret and A. Zehnacker-Rentien, Chem. Phys. Lett., 1994, 220, 235.

28 O.-H. Kwon, C. M. Othon, T. H. Yoo, J. A. Van Deventer, D. A. Tirrell and A. H. Zewail, Proc. Natl. Acad. Sci. U. S. A., 2010, 107, 17101 .

29 K. J. Laidler, Chemical Kinetics, Harper \& Row, New York, 1987.

30 E. Pines, D. Pines, T. Barak, B.-Z. Magnes, L. M. Tolbert and J. E. Haubrich, Ber. Bunsen-Ges. Phys. Chem., 1998, 102, 511.

31 N. Al-Lawatia, J. Husband, T. Steinbrecher and O. K. Abou-Zied, J. Phys. Chem. A, 2011, 115, 4195.

32 A. Bach and S. Leutwyler, Chem. Phys. Lett., 1999, 299, 381.

33 W.-H. Fang, J. Phys. Chem., 1999, 103, 5567.

34 O. F. Mohammed, O.-H. Kwon, C. M. Othon and A. H. Zewail, Angew. Chem., Int. Ed., 2009, 48, 6251.

35 Unlike electronic spectroscopy, IR spectroscopy has sensitivity to vibration providing bond-selective local information. Therefore, ultrafast IR spectroscopy poses to provide deep insight into the sequential movement of protons tracking specific vibrational marker modes, e.g., the transient IR-active absorption bands of hydrated proton and imine/amine (reactant/product) groups.

36 W. P. Jencks, Catalysis in Chemistry and Enzymology, Dover, New York, 1987.

37 B. J. Siwick and H. J. Bakker, J. Am. Chem. Soc., 2007, 129, 13412.

38 It has been reported that this process does not occur in the gas phase (see C. Tanner, M. Thut, A. Steinlin, C. Manca and S. Leutwyler, J. Phys. Chem. A, 2006, 110, 1758). This discrepancy can be explained based on different energetic and dynamic aspects in the condensed phase from those in the gas phase: higher water activity as a proton donor/acceptor; solvation to stabilize ionic species involved during the reaction; and thermal fluctuation of medium that helps geometric configurational optimization for proton tunneling in the solution phase $\mathrm{e}^{23,27}$. 